\title{
An Elementary Proof for a Class of Integral Inequality System
}

\author{
Xibing $\mathrm{He}^{1}$, Yupei Zhang ${ }^{2}$ \\ ${ }^{1}$ Department of Mathematics and Computer Science, Nanchang Normal University, Nanchang, \\ 330032, China \\ ${ }^{2}$ Nanchang Institute of Science \& Technology, Nanchang, 330108, China
}

Keywords: Gronwall-Bellman’s inequality, Integral inequality, Differential equation, Variable trans-formation method.

Abstract: The purpose of this paper is to establish some results for the class of system of integral inequalities by means of variable transformation method and Gronwall-Bellman's inequality. These results presented in this paper generalize and improve some recent results. Meanwhile, two examples indicate the effectiveness of the main results.

\section{Introduction}

It is well known that the differential and integral inequalities plays an important role in the study of quantitative properties and stability of solutions of differential and integral equations (see, e.g., [2, 3, 5, 6] and references therein). The celebrated Gronwall's inequality which was established in 1919 by Gronwall ${ }^{[4]}$ occurs very frequently in the theory of differential equations in various context. According to the different motivations, various inequalities and their generalized forms of Gronwall's inequality have been obtained and applied extensively in diversity areas including global existence, uniqueness, stability, boundary value problem, and other properties (see, e.g., [1-7] and references therein). In particular, Bellman [1] gave the Gronwall-Bellman's inequality as following:

Lemma $1^{[1,6]}$ (Gronwall-Bellman's inequality) suppose $\phi(t)$ and $b(t)$ are nonnegative continuous functions on $J=[\alpha, \beta]$. Assume that $\phi(t) \leq a+\int_{\alpha}^{t} b(s) \phi(s) d s, \forall t \in J$, where $a \geq 0$ is a constant. Then $\phi(t) \leq a e^{f_{\alpha}^{t} b(s) d s}, \forall t \in J$.

In 1963, Bellman and Cook [2] generalized the above inequality as following:

Lemma $2^{[2,5]}$ (Generalized Gronwall-Bellman's inequality) suppose $\phi(t)$ and $b(t)$ are nonnegative continuous functions on $J=[0, T)(T \leq+\infty)$. Assume that $c(t)>0$ is a monotone nondecreasing function on $J$. If $\phi(t) \leq c(t)+\int_{\alpha}^{t} b(s) \phi(s) d s, \forall t \in J$ holds, then $\phi(t) \leq c(t) e^{f_{\alpha}^{t} b(s) d s}, \forall t \in J$.

The purpose of this paper is to prove a class of integral inequality system by the generalized

Gronwall-Bellman's inequality and variable transformation method. Meanwhile, we shall give two examples as an application. These results presented in this paper generalize and improve some recent results of Xuerong Mao in $1989^{[6]}$.

\section{The Main results}

One main results are given in the following the theorems:

Theorem 1. Suppose $u(t), v(t)$ and $h_{i}(t)(i=1,2,3,4)$ are nonnegative continuous functions on $[0, \infty)$, and let $c_{j}(t)(j=1,2)$ be positive and monotone continuous function on $[0, \infty)$. Assume that there exists a nonnegative continuous function $\theta(t)$ such that $h_{i}(t) \leq \theta(t)(i=1,2,3,4)$ for any $t>0$. If the following inequalities (1) 


$$
u(t) \leq c_{1}(t)+\int_{0}^{t} h_{1}(s) v(s) e^{\mu s} d s+\int_{0}^{t} h_{3}(s) u(s) d s, v(t) \leq c_{2}(t)+\int_{0}^{t} h_{2}(s) u(s) e^{-\mu s} d s+\int_{0}^{t} h_{4}(s) v(s) d s
$$

hold on $[0, \infty)$, where $\mu \geq 0$ is a constant. Then there exist continuous functions $C(t)>0$ and $\rho_{k}(t) \geq 0(k=1,2)$ on $[0, \infty)$ such that $u(t) \leq C(t) e^{\int_{0}^{t} \rho_{1}(s) d s}, v(t) \leq C(t) e^{\int_{0}^{t} \rho_{2}(s) d s}$.

Proof First of all, let us make the transformation

$$
u(t)=\tilde{u}(t) e^{-\alpha t}, v(t)=\tilde{v}(t) e^{-\beta t}, t \in[0, \infty),
$$

where $\alpha, \beta$ satisfy $\alpha+\mu=\beta, \alpha>0$. Thus we substitute (2) into (1), and in view of $\alpha+\mu=\beta$, we obtain

$$
\tilde{u}(t) e^{-\alpha t} \leq c_{1}(t)+\int_{0}^{t}\left(h_{1}(s) \tilde{v}(s)+h_{3}(s) \tilde{u}(s)\right) e^{-\alpha s} d s, \tilde{v}(t) e^{-\beta t} \leq c_{2}(t)+\int_{0}^{t}\left(h_{2}(s) \tilde{u}(s)+h_{4}(s) \tilde{v}(s)\right) e^{-\beta s} d s
$$

Meanwhile, since $h_{i}(t) \leq \theta(t)(i=1,2,3,4)$ on $(0, \infty)$, we get

$$
\tilde{u}(t) \leq c_{1}(t) e^{\alpha t}+\int_{0}^{t}(\tilde{v}(s)+\tilde{u}(s)) \theta(s) e^{\alpha(t-s)} d s, \tilde{v}(t) \leq c_{1}(t) e^{\beta t}+\int_{0}^{t}(\tilde{v}(s)+\tilde{u}(s)) \theta(s) e^{\beta(t-s)} d s .
$$

Thus $\tilde{u}(t)+\tilde{v}(t) \leq c_{1}(t) e^{\alpha t}+c_{2}(t) e^{\beta t}+\int_{0}^{t}(\tilde{u}(s)+\tilde{v}(s)) \theta(s)\left(e^{\alpha(t-s)}+e^{\beta(t-s)}\right) d s$

$$
\leq\left(c_{1}(t)+c_{2}(t)\right) e^{\beta t}+\int_{0}^{t} 2 \theta(s)(\tilde{u}(s)+\tilde{v}(s)) e^{\beta(t-s)} d s, \forall t \geq 0 .
$$

Namely $(\tilde{u}(t)+\tilde{v}(t)) e^{-\beta t} \leq\left(c_{1}(t)+c_{2}(t)\right)+\int_{0}^{t} 2 \theta(s)(\tilde{u}(s)+\tilde{v}(s)) e^{-\beta s} d s, \forall t \geq 0$.

It follows from Lemma 2 that

$$
(\tilde{u}(t)+\tilde{v}(t)) e^{-\beta t} \leq C(t) e^{\int_{0}^{t} 2 \theta(s) d s}, \forall t \geq 0,
$$

where $C(t)=c_{1}(t)+c_{2}(t)$. Setting $\rho_{1}(t)=2 \theta(t)+\mu t, \rho_{2}(t)=2 \theta(t)$, it follows from (2) and (3) that we obtain the conclusion of the Theorem 1 . This completes the proof.

Example 1 Consider the following initial-value problem

$$
\left\{\begin{array}{l}
u^{\prime}(t) \leq 4 t+1+2^{-t} v(t) e^{2 t}+(\sqrt{t}+1) u(t), \forall t \in[0,+\infty) \\
v^{\prime}(t) \leq 2^{t} \ln 2+\left(1+t^{2}\right)^{-1} u(t) e^{-2 t}+\left(1+3^{-1} t\right) v(t), \forall t \in[0,+\infty) \\
u(0)=1, v(0)=\ln 2
\end{array}\right.
$$

where $h_{1}(t)=2^{-t}, h_{2}(t)=\left(1+t^{2}\right)^{-1}, h_{3}(t)=\sqrt{t}+1, h_{4}(t)=1+3^{-1} t$. Set $\theta(t)=\sqrt{t}+2$. Then we know that $0 \leq h_{i}(t) \leq \theta(t)(i=1,2,3,4)$ for $\forall t \in[0, \infty)$. Hence, by a direct calculation and using Theorem 1 , it can be easily shown that the solutions $(u(t), v(t))$ of system (4) have a pair of priori upper control functions respectively in the following form: $u(t) \leq\left(2 t^{2}+t+2^{t}\right) e^{\frac{4}{3} \sqrt{3 t^{3}}+2 t+t^{2}}$, $v(t) \leq\left(2 t^{2}+t+2^{t}\right) e^{\frac{4}{3} \sqrt{3 t^{3}}+2 t}, \forall t \in[0,+\infty)$.

If $\mathrm{h}_{3}(\mathrm{t}) \equiv 0 \equiv \mathrm{h}_{4}(\mathrm{t})$ on $[0, \infty)$ in Theorem 1 , then we have the following corollary:

Corollary 1 Let $u(t), v(t), h_{i}(t)(i=1,2,3,4)$ and $c_{j}(t)(j=1,2)$ be same as Theorem 1 . If the

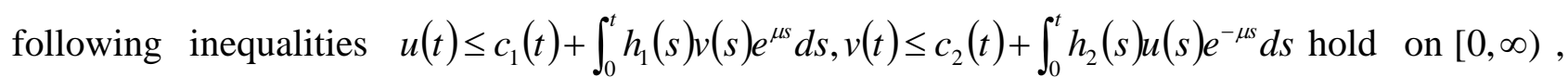
where $\mu \geq 0$ is a constant. Then there exist continuous functions $C(t)>0$ and $\rho_{k}(t) \geq 0(k=1,2)$ on $[0, \infty)$ such that $u(t) \leq C(t) e^{\int_{0}^{t} \rho_{1}(s) d s}, v(t) \leq C(t) e^{\int_{0}^{t} \rho_{2}(s) d s}$.

The following theorem shows some structure of solutions in the case when $c_{i}(t) \equiv c_{i} \geq 0(i=1,2)$ 
on $[0, \infty)$ in Theorem 1 .

Theorem 2 Let $u(t), v(t), h_{i}(t)(i=1,2,3,4)$ and $\theta(t)$ be same as Theorem 1. If the following inequalities

$$
u(t) \leq c_{1}+\int_{0}^{t} h_{1}(s) v(s) e^{\mu s} d s+\int_{0}^{t} h_{3}(s) u(s) d s, v(t) \leq c_{2}+\int_{0}^{t} h_{2}(s) u(s) e^{-\mu s} d s \quad+\int_{0}^{t} h_{4}(s) v(s) d s \quad \text { hold, }
$$

where $c_{1}, c_{2}, \mu$ are nonnegative constants. Then there exist a constant $M>0$ and continuous functions $\rho_{k}(t) \geq 0(k=1,2)$ on $[0, \infty)$ such that $u(t) \leq M e^{\int_{0}^{t} \rho_{1}(s) d s}, v(t) \leq M e^{\int_{0}^{t} \rho_{2}(s) d s}$.

Proof If we make the transform (2) and use Lemma 1, then the following proof is the same as Theorem 1, we omit the proof. This completes the proof.

Remark 1. If we set $\theta(t) \equiv M>0$ on $[0, \infty)$ in Theorem 2, it is easy to see that our result extends the conclusion of Xuerong Mao in 1989 [6].

If $c_{i}(t) \equiv c_{i} \geq 0(i=1,2)$ on $[0, \infty)$ in Corollary 1 or $h_{3}(t) \equiv h_{4}(t) \equiv 0$ on $[0, \infty)$ in Theorem 2 , then we have the following corollary:

Corollary 2. Let $u(t), v(t), h_{i}(t)(i=1,2,3,4)$ and $\theta(t)$ be same as Theorem 1 . If

$$
u(t) \leq c_{1}+\int_{0}^{t} h_{1}(s) v(s) e^{\mu s} d s, v(t) \leq c_{2}+\int_{0}^{t} h_{2}(s) u(s) e^{-\mu s} d s
$$

hold on $[0, \infty)$, where $c_{1}, c_{2}, \mu$ are nonnegative constant. Then there exist a constant $M>0$ and nonnegative continuous functions $\rho_{k}(t) \geq 0(k=1,2)$ on $[0, \infty)$, such that $u(t) \leq M e^{\int_{0}^{t} \rho_{1}(s) d s}, v(t) \leq M e^{\int_{0}^{t} \rho_{2}(s) d s}$.

Example 2. Consider the following initial-value problem

$$
\left\{\begin{array}{l}
u^{\prime}(t) \leq\left(3^{-2 t}+1\right) v(t) e^{4 t}, \forall t \in[0,+\infty) \\
v^{\prime}(t) \leq\left(1+\sqrt{1-2^{-t}}\right) u(t) e^{-4 t}, \forall t \in[0,+\infty) \\
u(0)=3, v(0)=1
\end{array}\right.
$$

It is easy to see that $1 \leq h_{1}(t)=3^{-2 t}+1 \leq 2$ and $1 \leq h_{2}(t)=1+\sqrt{1-2^{-t}} \leq 2$ for $\forall t \in(0,+\infty)$. Let $\theta(t)=2, \forall t \in[0,+\infty)$. By a direct calculation and using Corollary 2, we can easily show that the solutions $(u(t), v(t))$ of system (5) have a pair of priori upper control functions respectively in the following form: $u(t) \leq 4 e^{4 t+2 t^{2}}, v(t) \leq 4 e^{4 t}, \forall t \in[0,+\infty)$.

\section{Acknowledgements}

In this paper, the research was supported by Science and Technology Research Project of Jiangxi Provincial Department of Education (No. GJJ151250, GJJ161244), Startup Foundation for Doctors of Nanchang Normal University (No. 2015027, 2015026), Nature Science Foundation of China (No. 11661057).

\section{References}

[1] Bellman R. The Stability of differential equations [J]. Duke Mathematical Journal, 1953, 10: 643-647.

[2] Bellman R, Cook K L. Differential-difference equations (Mathematical Science and Engineering, Vol. 6) [M]. New York, London: Academic Press, 1963.

[3] Dragomir S S. Some Gronwall type inequalities and applications [M]. New York: Nova Science Publishers, 2003. 
[4] Gronwall T H. Note on the derivatives with respect to a parameter of the solutions of differential equations [J]. Annals of Mathematics, 1919, 20(2): 293-296.

[5] Hale J K. Ordinary differential equations [M]. New York: Wiley-Interscience, 1969.

[6] Kuang J C. Applied Inequalities (4.ed) (Chinese) [M]. Ji’nan: Shandong Science and technology Press, 2010.

[7] Reddy K N. Integral inequalities and applications [J]. Bulletin of the Australian Mathematical Society, 1980, 21(1): 13-20. 